Relations industrielles

Industrial Relations

\title{
Economics of Labor Relations. By Gordon F. Bloom and Herbert R. Northrup. Fifth Edition. Richard D. Irwin, Inc., Homewood, Illinois, 1965, 911 pp.
}

\section{Gérard Dion}

Volume 20, numéro 2, 1965

URI : https://id.erudit.org/iderudit/027573ar

DOI : https://doi.org/10.7202/027573ar

Aller au sommaire du numéro

Éditeur(s)

Département des relations industrielles de l'Université Laval

ISSN

0034-379X (imprimé)

1703-8138 (numérique)

Découvrir la revue

Citer ce compte rendu

Dion, G. (1965). Compte rendu de [Economics of Labor Relations. By Gordon F. Bloom and Herbert R. Northrup. Fifth Edition. Richard D. Irwin, Inc.,

Homewood, Illinois, 1965, 911 pp.] Relations industrielles / Industrial Relations, 20(2), 397-397. https://doi.org/10.7202/027573ar

Tous droits réservés @ Département des relations industrielles de l'Université Laval, 1965
Ce document est protégé par la loi sur le droit d'auteur. L’utilisation des services d'Érudit (y compris la reproduction) est assujettie à sa politique d'utilisation que vous pouvez consulter en ligne.

https://apropos.erudit.org/fr/usagers/politique-dutilisation/ 
1-La définition de la planification en longue période.

2-Les principes majeurs concernant l'organisation qu'il faut mettre sur pied en vue $d^{\prime} u n e$ planification fructueuse.

3-La démarche à suivre dans l'édification d'un plan.

4-La relation entre les objectifs de l'entreprise et le plan.

5--La détermination de la période couverte par le plan.

6-L'utilisation des techniques quantitatives en vue d'améliorer le processus et les résultats.

7-Les problèmes majeurs auxquels fait face actuellement la planification.

Ceux qui prirent part à la discussion tombèrent d'accord sur la nécessité de la planification et de la coordination des plans de courte période avec ceux de longue-période. La flexibilité et la créativité sont deux conditions essentielles dans l'élaboration d'un plan. L'expérience démontre que des plans couvrant une période de cinq ans sont les plus pratiques.

Co volume apporte un complément valable oux principes et aux expériences pratiques qui sont présentées dans les manuels en administration des entreprises.

\section{Laurent BELANGER}

Economics of Labor Relations. By Gordon F. Bloom and Herbert R. Northrup. Fifth edition. Richard D. Irwin, Inc., Homewood, Illinois, 1965, 911 pp.

Cet ouvrage connait une cinquième édition cans l'espace de quinze ans. C'est déjà un signe manifestant la valeur d'un tel monuel.

Les auteurs l'ont mis à jour en tenant compte des développements dans les relations du travail de même que des nouveaux problèmes qui ont surgi depuis quelques années: The Civil Rights Act de 1964, le chômage, la reconversion industrielle, les nouvelles formules de négociation collective; le plan Kaiser, etc.

Le dernier chapitre est consacré aux problèmes du travail dans les années soixante: sécurité sociale, nécessité de nouveaux concepts, intervention gouvernementale, nécessité de redéfinir le rôle des syndicats et de la direction des entreprises comme celui de l'industrie et du gouvernement.
Ce chapitre se borne seulement à poser les problèmes dans des termes assez ternes et n'apporte rien de bien substantiel. Les auteurs ne semblent point pouvoir se dégager de conceptions dépassées qu'ils reprochent, avec raison, au syndicalisme ouvrier.

Entre les mains de professeurs dynamiques et informés, ce manuel peut être un bon instrument. A la fin de chaque chapitre on propose des questions pour discussions et on présente une bibliographie sélective utile à l'étudiant pour compléter ses études.

\section{Gérard DION}

Readings on Economic Sociology, Edited by Neil J. Smelzer, Readings in Modern Sociology Series, Prentice-Hall, Inc., Englewood Cliffs, New Jersey, 1965, 303 pages.

\section{The Social Context of Economic Behavior,} par W.T. Tucker Holt, Rinehart and Winston, Inc., 1964, 163 pages.

Bien que la sociologie et l'économique possèdent une certaine autonomie sur le plan des concepts, des hypothèses et de la démarche intellectuelle, on ne peut pour autant nier l'existence des points de rencontre entre ces deux disciplines.

Ces deux auteurs se sont assignés la tâche de faire ressortir ces points en montrant la façon dont le comportement économique s'insère dans un contexte social beaucoup plus vaste où des facteurs culturels, psychologiques, organisationnels, politiques, viennent s'ajouter aux variables économiques pour arriver à une meilleure explication de la réalité sociale.

\section{Variables culturelles et activité économique}

Pour cette première section, Smelzer a fait un choix de lecteurs qui mettent à jour les relations entre la structure économique et la vie culturelle de la société. En effet, un premier article, celui de Birnbaum, examine de nouveau les théories de Marx et de Weber sur I'origine du capitalisme. Dans un autre article, Bendix retrace brièvement les changements dans le contenu des idéologies anglo-américaines et russes, la signification historique des idéologies directoriales, les différences entre les formes totalitaires et non-totalitaires de la subordination dans la société industrielle.

Le traitement des variables culturelles chez Tucker se situe à un niveau beaucoup plus concret. L'activité économique influence la culture d'une société et vice-versa. Par 